\title{
Research on rapid process optimization technology based on Improved Genetic Algorithm
}

\author{
Hang Yu*, Liqin Miao, Jichun Jiang, Heping Jiang , Wanrui Cui, \\ Fanjun Meng, Lijun Wang, Yuxin Li, Xiaojiao Gao and Yue Fan \\ Department of numerical design\&manufacture
}

Changchun Research Institute of Equipment and Technology, Changchun

\author{
130012,P.R.CHINA \\ yuhang9511@163.com
}

\begin{abstract}
Keywords: Improved genetic algorithm Process path Optimization Adaptive crossver Adaptive mutation

Abstract. In this paper, the vehicle engine block processing characteristics was analyzed based on body machining process path problem, process route optimization mathematical model was established, the principle of the basic genetic algorithm and genetic operation were analyzed also, a kind of improved genetic algorithm was proposed in the paper. The optimal strategy under improved genetic algorithm was designed, which could be used to optimize the engine block machining process path, solve in industry and trade group for the shortest machining path search question, and specific examples could be used to validate the effectiveness.
\end{abstract}

\section{Introduction}

The machining process of engine body is complicated, there are 16 faces need to be manufactured, according to the manufacturing characteristic, machining method, tool type and tool size, the faces were classified, and working steps constitute group, the tool installed one time could finish manufacture all the characteristics. How to optimize the machining path of working step group has become the base which could improve the machining efficiency of engine body and improve the optimization of process schemes. Genetic algorithm which is global search algorithm, the searching efficiency is very high, and the process is very simple. In this paper, under the standard genetic algorithm, through the improvement of genetic algorithm, the optimization of shortest path for the engine body was accomplished.

\section{Description of rapid process optimization for an engine body}

According to the characteristics of engine block parts, the process design of process planning and optimization system should be followed:

1) First machining orientation feature, the hole feature of the process;

2) Advanced line of rough machining, and then semi finishing or finishing;

3 ) According to the drilling center hole, drilling or milling, boring, reaming hole;

4) In accordance with the first tapping drill center hole, after drilling;

5) Taking into account the special nature of the large box part, the basic should be followed by the first of all the characteristics of a bearing surface after the processing is completed and then transposition.

In order to describe the working process of the engine block, the machining path is described as: Distance between the known $\mathrm{N}$ working steps,Starting from a working position. In turn processing n-1 working steps.Each working step must be processed and processed only once,From the beginning of the first working step to the last working position, the path of the tool path is the working path. How to arrange the processing sequence of the $\mathrm{n}$ points in a plane.Making the tool through all the process of 
the point of the shortest path is the key to achieve the engine body 16 plane rapid process preparation. It is a typical combination optimization problem to find the shortest path problem in the process of machining the $\mathrm{N}$ position. The objective function can be as shown in Eq. 1.

$$
Z=\sum_{i=0}^{\mathrm{n}-1} d\left(V_{i}, V_{i+1}\right)
$$

Among them, $V_{i}$ indicates the processing position of the $I, V_{0}$ Express origin, $d\left(V_{i}, V_{i+1}\right)$ Indicates the distance between the position $V_{i}$ to the point $V_{i+1}$ 。

\section{Improved genetic algorithm design for the process route optimization of an engine}

Genetic algorithm is a kind of stochastic optimization algorithm, which is based on the genetic evolution mechanism in nature[2]. The genetic algorithm is simple and efficient. Optimization problem for solving process path optimization. Genetic algorithms are generally performed by selectin, crossover and mutation operations. But for solving the problem of combinatorial optimization problem in space, The parameters of the algorithm and the design of fitness function are not reasonable. Even if the algorithm is trapped in local optimal solution or search time is too long, and so on.

In order to complete the optimization of engine block machining process path. The standard genetic algorithm is improved in this paper.The improved genetic algorithm is used to optimize the shortest path of multiple position points on a plane. In order to avoid the phenomenon of premature and random roaming in genetic algorithm. The fitness, crossover probability and mutation probability of genetic algorithm are improved. And the optimal solution is not lost through the guarantee of the optimal strategy. The optimization performance of genetic algorithm is improved. Genetic algorithm design is as follows:

\section{1) Coding method}

Permutation encoding method,. The working steps of each working step corresponding to each working step corresponding to the location of the use of the only natural number (such as $2,1,3 \ldots \mathrm{N}$ ), the length of the gene is equal to the total number of steps in the process. Take a sequence of all working steps in the working group as a chromosome. Define each position corresponding to a gene as a gene. Decoding can be directly based on the chromosome decoding, Convenient fitness value calculation. Such as gene string: 2457136 , Represents the working path for the working group: 2->4->5->7->1->3->6, Working step Total 7 .

\section{2) Selection of initial population}

The effect of the number of 100 chromosomes in the initial population is better in the experiment. 200 chromosomes were generated randomly in order to change the gene sequence in the chromosome. The fitness value of each chromosome was calculated.The 100 individuals with better fitness values were used as the initial population.

\section{3 ) Fitness function}

For improving the performance of genetic algorithm, As far as possible to avoid premature and random roaming phenomenon, It is often necessary to determine the fitness function by amplifying or reducing the target function. The scaling of the objective function is called the adaptive scale transformation. The objective function is to design the fitness function as shown in Eq. 2.

$$
\mathrm{F}=\mathrm{b} / \sqrt{Z}
$$

$\mathrm{Eq} 2, \mathrm{Z}$ is Representing the target function value of the shortest path in the working group. $\mathrm{b}$ is constant, The search results of the $b=20$ algorithm are better by the experiment.

\section{4) Choice}

Using roulette method to select breeding individuals. In order to avoid the loss of the optimized individuals in the evolution, the generation gap is used to control the proportion of the population in each generation, Through experiment test, The generation gap is set as the $90 \%$ algorithm search efficiency is higher. The method of selection is, In each population, $90 \%$ of the individuals were 
selected for genetic manipulation. $10 \%$ the best individual in the parent generation directly into the sub generation, In order to ensure that the good individual in the original population will not be lost because of evolution[3].

\section{5) Adaptive crossover}

Although GA is effective for solving complex problem, it is easy to produce premature convergence, By studying the operating parameters of GA, Pc selection of cross probability, To facilitate the generation of new individuals, But it is easy to destroy the good individual in the population. And the choice is too small, Is not conducive to the generation of new individuals, the impact of the problem is further solved, it is difficult to find the optimal solution. Therefore, the crossover probability $\mathrm{Pc}$ is designed according to the adaptive degree of individual in each generation. Adaptive crossover probability Pc as shown in Eq. 3.

$$
\mathrm{P}_{\mathrm{c}}= \begin{cases}\mathrm{k}_{1} & f_{c} \leq f_{\text {avg }} \\ \left.k_{1}-\frac{k_{2}\left(f_{c}-f_{\text {avg }}\right)}{\left.f_{\max }-f_{\text {avg }}\right)}\right) & f_{c}>f_{\text {avg }}\end{cases}
$$

Formula fe is a large fitness value for the two chromosomes waiting to cross, favg is the average fitness value of the current population, fmax is the maximum fitness value of chromosome in the population. $\mathrm{k} 1$ and $\mathrm{k} 2$ is Constant, $\mathrm{K} 1$ is set to $0.5, \mathrm{~K} 2$ is set to 0.9 . The adaptive adjustment of Pc and the degree of convergence of the algorithm to the reverse, thus effectively prevent the algorithm's early convergence, improve the algorithm's search efficiency.

Cross method using a little cross method. That is, a crossover site randomly selected from the parent chromosome A1 and A2. A1 cross position and the right side of the gene value as a sub chromosome A1 'corresponding position of the gene value, These genes were randomly deleted from the other parent chromosome A2; Then, A2 gene from left to right and left to right in the blank space of A1. The same can be obtained from the sub chromosome A2 '. Examples as shown in Fig. 1.

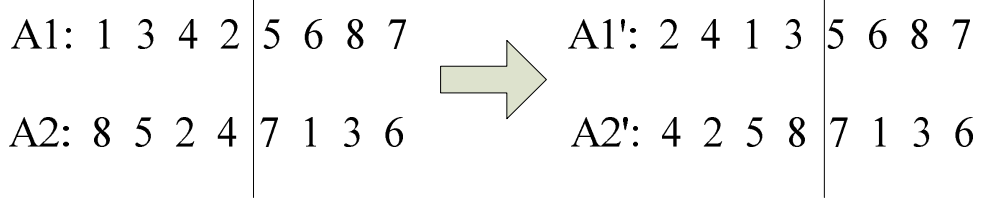

Fig. 1 Cross operation example

\section{6) Adaptive mutation}

In this paper, the adaptive mutation probability is used to improve the performance of the algorithm. According to the adaptive degree of individual in each generation of the population, the adaptive mutation probability Pm as shown in Eq. 4.

$$
\mathrm{P}_{\mathrm{m}}= \begin{cases}\mathrm{k}_{1} & f_{m} \leq f_{\text {avg }} \\ k_{1}\left(1-\frac{f_{m}-f_{\text {avg }}}{f_{\max }-f_{\text {avg }}}\right) & f_{m}>f_{\text {avg }}\end{cases}
$$

The fm is waiting for chromosome fitness value, favg is the population average fitness values, fmax is the chromosome in the population with the maximum fitness value, $\mathrm{k} 1$ is a constant, where $\mathrm{k} 1$ is set to 0.1 . The adaptive adjustment of Pm and the degree of convergence of the algorithm to the reverse, thus effectively prevent the algorithm's early convergence, improve the algorithm's search efficiency.

\section{7) Optimal strategy}

Since the generation gap is $90 \%$, the initial population number is 100 , and the number of chromosomes involved in the crossover and mutation is $100 * 90 \%=90$. When the crossover and mutation will generate offspring chromosome number 90 . In order to keep the balance of the parent 
and offspring of the chromosome number, the parent population adaptation degree value higher 10 $(100 *(1-90 \%)=10)$ chromosomes selection directly into the offspring, which can ensure parent in the better part of chromosome will not because of the randomness of evolution and lost.

By keeping the best individual in the process of evolution as the best individual, the method prevents the optimal individual in the process of evolution. The fitness value of the optimal individual is compared with the fitness value of the elite individual, and the fitness value is greater than that of the individual[4], Then the essence of the individual will be replaced by the best individual in the quilt, otherwise the essence of the individual remains unchanged[5]. By the essence of the individual, it can be saved to the optimal individual in the process of the generation of multiple generations so far. Engine block machining process path optimization algorithm based on the improved genetic algorithm as shown in Fig. 2.

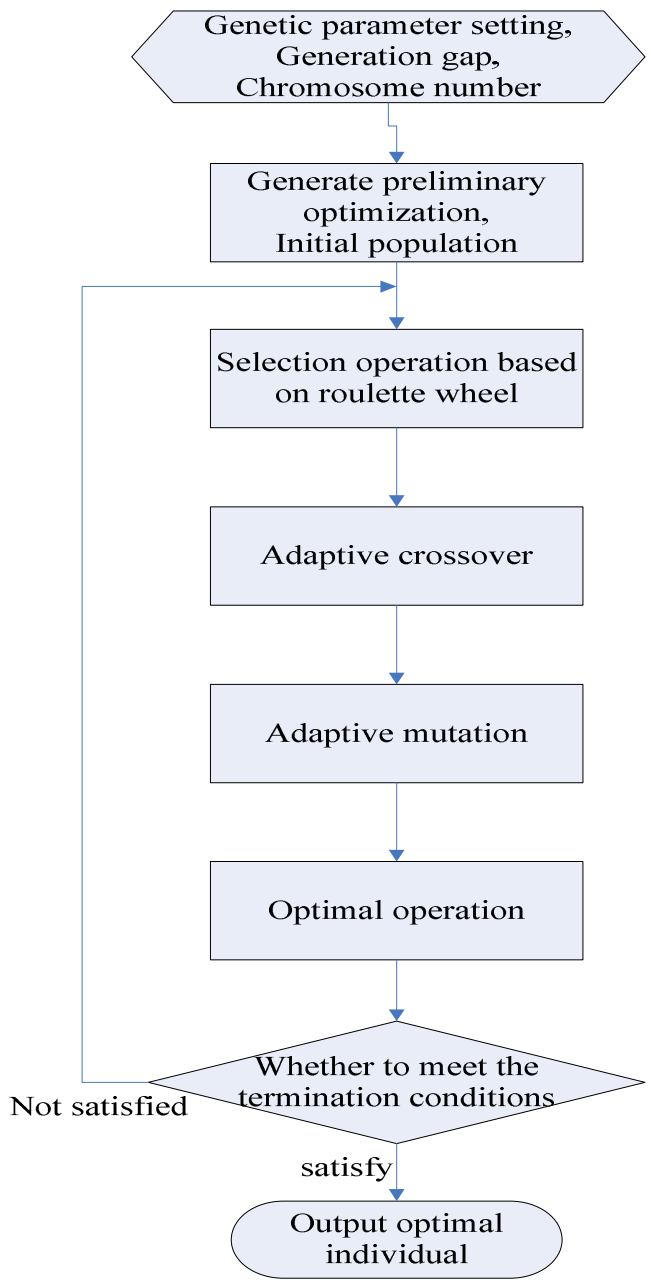

Fig. 2 The process of engine block machining process path optimization

\section{Algorithm verification}

In this paper, the improved genetic algorithm based on the upper section of the engine body is proposed, that is, the process route of the engine body is made, that is, the characteristics of the processing sequence is selected in each working step. Taking the screw hole machining working steps of the engine block, the working steps are 22 steps, and the engine block is shown in Fig. 3. 


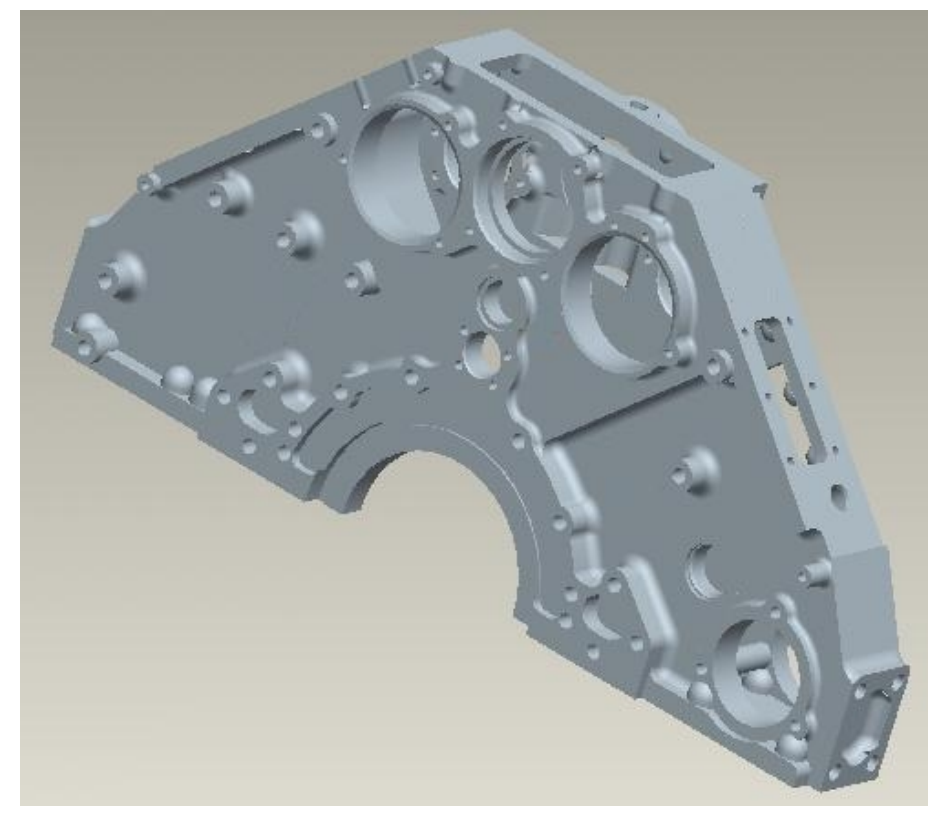

Fig. 3 engine block drive end

Each working step is completed a hole machining, the hole processing position coordinates as shown in Table 1, need to determine the cutting tool path to complete all 22 holes machining.

Table 1 processing position coordinates of the screw holes $(\mathrm{mm})$

\begin{tabular}{|c|c|c|c|c|c|}
\hline $\begin{array}{c}\text { Hole } \\
\text { position } \\
\text { number }\end{array}$ & $\begin{array}{c}\mathbf{X} \\
\text { coordinates }\end{array}$ & $\begin{array}{c}\mathbf{Y} \\
\text { coordinates }\end{array}$ & $\begin{array}{c}\text { Hole } \\
\text { position } \\
\text { number }\end{array}$ & $\begin{array}{c}\mathbf{X} \\
\text { coordinates }\end{array}$ & $\begin{array}{c}\mathbf{Y} \\
\text { coordinates }\end{array}$ \\
\hline $\mathbf{1}$ & -300 & 12 & $\mathbf{1 2}$ & 126 & 24 \\
\hline $\mathbf{2}$ & -300 & 66 & $\mathbf{1 3}$ & 126 & 52 \\
\hline $\mathbf{3}$ & -240 & 165 & $\mathbf{1 4}$ & 40 & 87 \\
\hline $\mathbf{4}$ & -180 & 252 & $\mathbf{1 5}$ & 48 & 126 \\
\hline $\mathbf{5}$ & -180 & 165 & $\mathbf{1 6}$ & -48 & 126 \\
\hline $\mathbf{6}$ & -120 & 165 & $\mathbf{1 7}$ & -40 & 87 \\
\hline $\mathbf{7}$ & 180 & 252 & $\mathbf{1 8}$ & -126 & 52 \\
\hline $\mathbf{8}$ & 180 & 165 & $\mathbf{1 9}$ & -126 & 24 \\
\hline $\mathbf{9}$ & 185 & 36 & $\mathbf{2 0}$ & -153 & 15 \\
\hline $\mathbf{1 0}$ & 153 & 69 & $\mathbf{2 1}$ & -185 & 36 \\
\hline $\mathbf{1 1}$ & 153 & 15 & $\mathbf{2 2}$ & -153 & 69 \\
\hline
\end{tabular}

Using the improved genetic algorithm, the process path optimization software is developed by Matlab. The parameters of genetic algorithm were set as the population size of 200, Each hole as a gene, With the position of hole said chromosome dot value, the length of each chromosome was 22 . Cutting tool to complete the machining sequence of all the holes in the plane to represent a chromosome. The adaptive crossover probability and adaptive mutation probability Eq. 3 and Eq. 4 are adopted, The generation gap is set to 0.9 , and the 1000 generation of evolution. Run the program in the ninety-fifth generation to get 22 hole processing steps of the shortest Process path is

"1->2->3->4->5->6->22->21->20->19->18->17->16->15->14->13->12->11->10->9->8->7"", The shortest path length as shown in Fig. 4. 


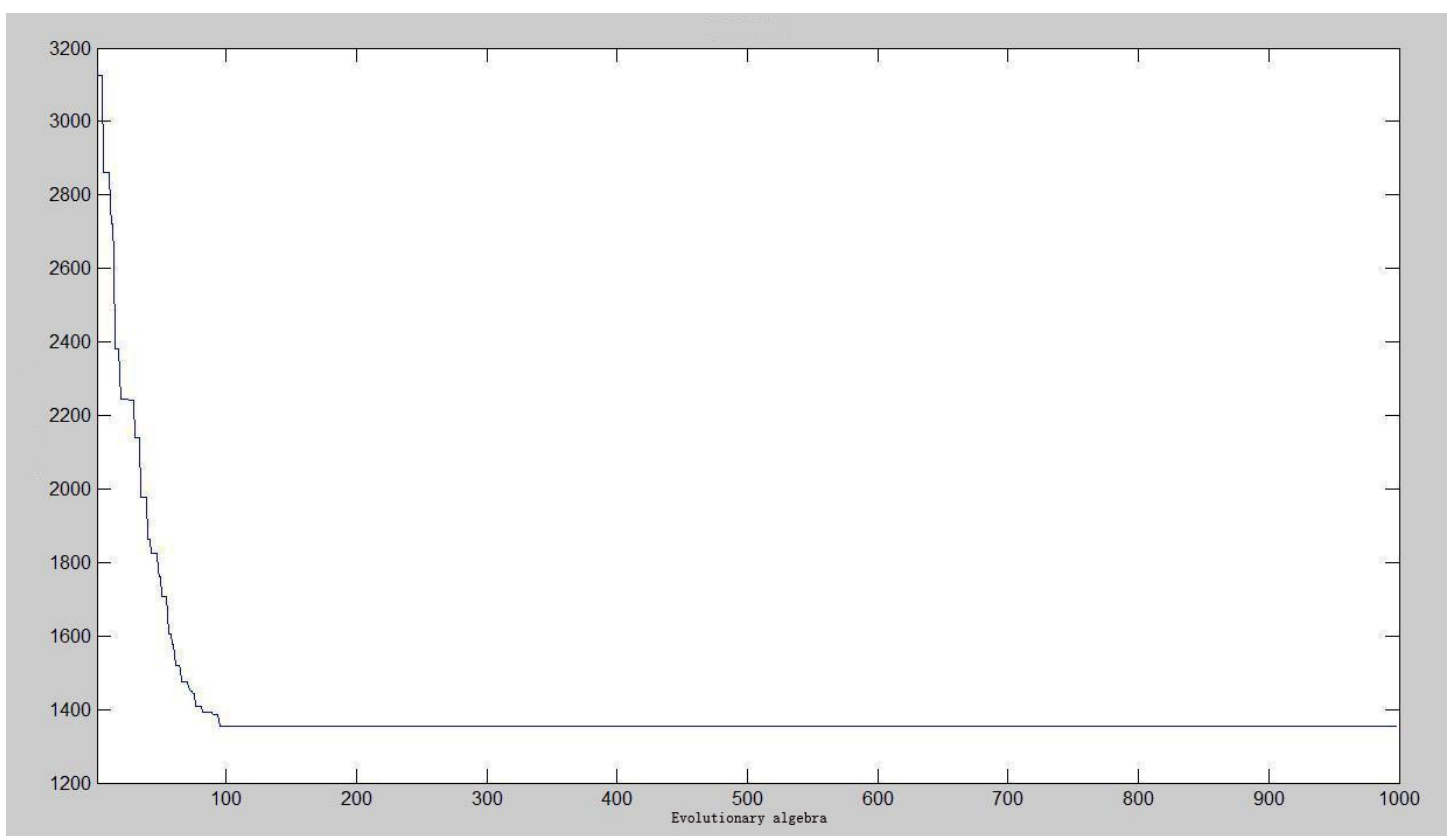

Fig. 4 optimization process of screw hole process path based on Improved Genetic Algorithm

Through genetic evolution, when running to the ninety-fifth generation, the process route of the working steps to achieve the shortest, The target value is reduced from 3138 to 1353, From Fig. 4 it can be seen that the improved genetic algorithm proposed in this paper is convergent. The algorithm converges to the optimal solution of 1353, and the evolutionary efficiency is higher than 95 generations. Optimal solution of the cutting tool path as shown in Fig. 5.

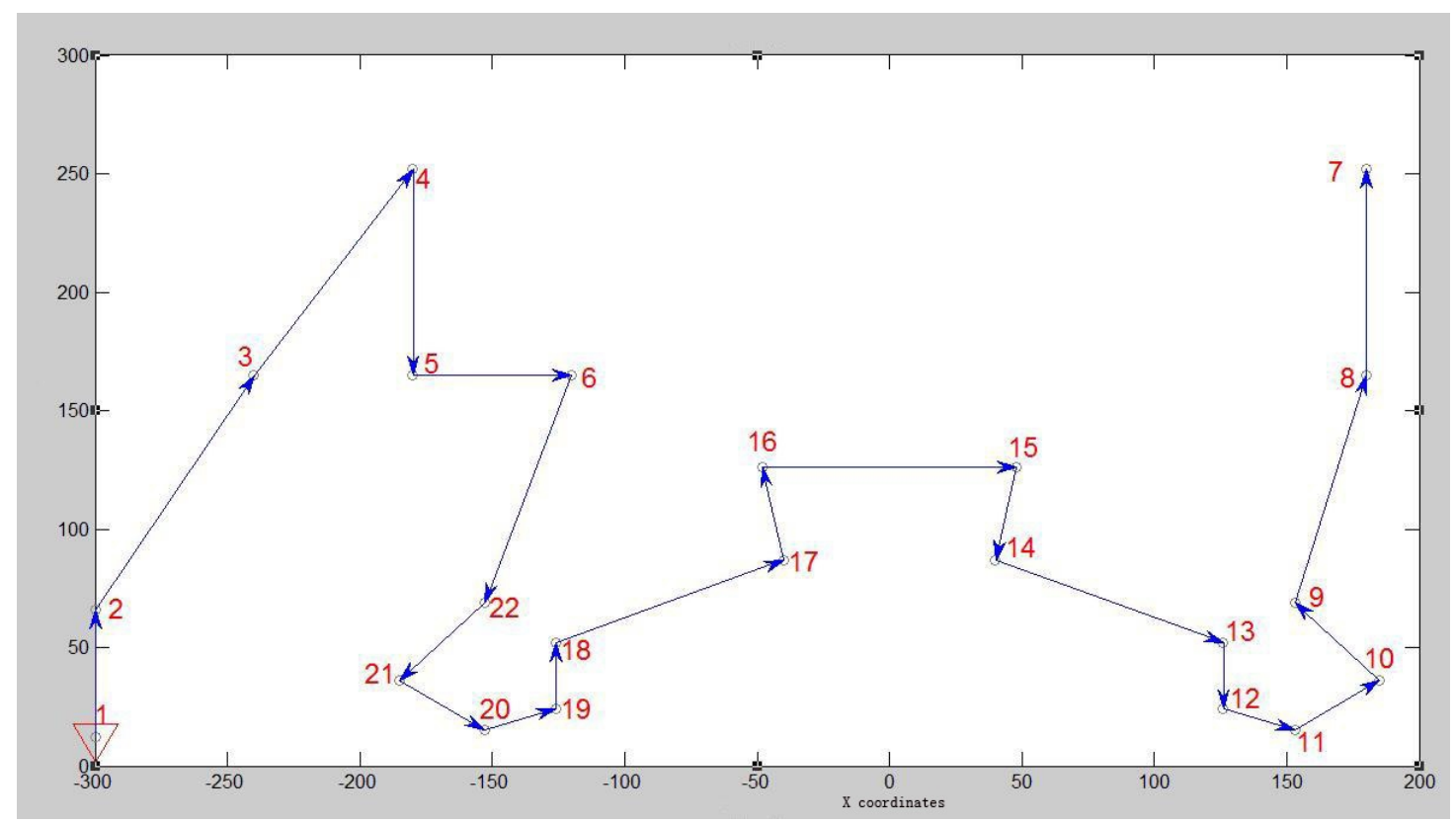

Fig. 5 optimal process route chart of thread hole machining

By Fig. 5 each machining hole and position are 1, 2, 3... 22 express, With the arrow line cutter working sequence representation, the first processing nock position of the hole, and then processing the corresponding position of the arrow holes. Surrounded by a red triangle hole represents the first processing hole, process route of the first step to processing hole location of the dot. The optimal solution of the cutting tool is the process of walking, Tool from the first hole processing, passes through the hole2, hole 3, hole4, hole 5, hole 6, 22 holes, 21 hole, hole 20, 19 holes, hole 18, 17 hole, hole 16, 15 hole, hole 14, 13 holes, hole 12, 11 holes, holes, 9 holes, hole 8, a hole 7. After processing the hole 7 , the body screw hole processing end. 


\section{Conclusion}

The machining characteristics of engine body was analyzed, the machining process path was talked about, the process path optimization mathematic model was established. The improved genetic algorithm was raised based on the analysis of genetic algorithm principle and genetic operation. The machining process path also was optimized by using the improved the genetic algorithm, the shortest path searching question in one working step group was accomplished. The coding, initial population, fitness function, select, adaptive crossover and adaptive mutation, quality protection strategy design, the efficiency was verified by specific example.

\section{References}

[1] Guobin Gong. Tool path planning and G/M code generation based on STEP-NC [D]. Shenyang University of Science and Technology.2011.In Chinese.

[2] KanDai. DNA Genetic algorithm and application in chemical processing[D].

ZheJiang University.2012.In Chinese.

[3] Sinan Tao. One improved genetic algorithm and application in workshop planning[D].

ChongQing University.2010.In Chinese.

[4] Xiaoli Liang. Application of genetic algorithm in intellectual paper generation[D].

ChangChun University of Science and Technology.2010.In Chinese.

[5] YanFeng. Research on multi-path planning based on cluster genetic algorithm[D].

Haerbin University of engineering.2009.In Chinese. 